\title{
Perfil de crianças em idade escolar submetidas a cirurgia em um hospital público
}

Profile of school-age children undergoing surgery at a public hospital

Perfil de niños en edad escolar sometidos a cirugía en un hospital público

Raquel Pan ${ }^{1}$ (D) https://orcid.org/0000-0003-1312-3889

Fernanda Accioly Tripode ${ }^{1}$ (D) https://orcid.org/0000-0001-9026-2239

Sérgio Antônio Zullo ${ }^{1}$ (D) https://orcid.org/0000-0002-7703-3172

Júnia Lanny Sousa Silva ${ }^{1}$ (D) https://orcid.org/0000-0002-5223-8147

Nazaré Pellizzetti Szymaniak ${ }^{1}$ (D) https://orcid.org/0000-0002-6825-6817

\section{Resumo}

Objetivo: Caracterizar crianças em idade escolar submetidas a intervenção cirúrgica em um hospital público do interior de Minas Gerais.

Métodos: Estudo descritivo e retrospectivo de abordagem quantitativa. Os dados de crianças em idade escolar, submetidas a procedimento cirúrgico no período de 2017 a 2019, foram obtidos no hospital de estudo. As variáveis estudadas foram sexo, idade, tipo e número do procedimento cirúrgico, assim como sua natureza, especialidade, município de residência, data do procedimento e destino após cirurgia. Análise estatística descritiva realizada para todas as variáveis.

Resultados: Foram identificados 825 pacientes pediátricos, sendo 549 (66,6\%) do sexo masculino, e a realização de 1.127 cirurgias. A especialidade com maior número de cirurgias foi Ortopedia e Traumatologia (34,2\%).

Conclusão: Constatou-se um perfil sociodemográfico de maior ocorrência de cirurgia em crianças com menor idade e do sexo masculino, submetidas a procedimento cirúrgico da especialidade de Ortopedia e Traumatologia, especialmente de urgência e emergência.

\begin{abstract}
Objective: To characterize school-age children who underwent surgical intervention in a public hospital in the countryside of Minas Gerais.

Methods: Descriptive and retrospective study with a quantitative approach. Data from school-age children who underwent a surgical procedure from 2017 to 2019 were taken from the study hospital. The variables studied were sex, age, type and number of the surgical procedure, as well as its nature, specialty, city of residence, date of procedure and destination after surgery. Descriptive statistical analysis performed for all variables.

Results: A total of 825 pediatric patients were identified, $549(66.6 \%)$ of whom were male and 1,127 surgeries were performed during this period. The specialty with the largest number of surgeries was Orthopedics and Traumatology (34.2\%).

Conclusion: There was a sociodemographic profile of greater occurrence of surgery in younger and male children undergoing surgical procedures in the specialty of Orthopedics and Traumatology, especially urgent and emergency procedures.
\end{abstract}

\section{Resumen}

Objetivo: Caracterizar a los niños en edad escolar que fueron sometidos a intervención quirúrgica.

Métodos: Estudio descriptivo y retrospectivo con abordaje cuantitativo. Los datos de los niños en edad escolar que se sometieron a un procedimiento quirúrgico en el período de 2017 a 2019 se obtuvieron del hospital de estudio. Las variables estudiadas fueron sexo, edad, tipo y número del procedimiento quirúrgico, así como su naturaleza, especialidad, municipio de residencia, fecha de procedimiento y destino después de la cirugía. Análisis estadístico descriptivo realizado para todas las variables.

Resultados: Se identificaron 825 pacientes pediátricos, 549 (66,6\%) eran varones y realizado 1.127 cirugías. La especialidad con mayor número de cirugías fue Ortopedia y Traumatología (34,2\%).

Conclusión: Hubo un perfil sociodemográfico de mayor ocurrencia de cirugías en niños de menor edad y de los varones sometidos al procedimiento quirúrgico de la especialidad de Ortopedia y Traumatología, especialmente de urgencia y emergencia.

\section{Descritores}

Procedimentos cirúrgicos; Perfil de saúde; Enfermagem pediátrica

\section{Keywords}

Surgical procedures; Health profile; Pediatric nursing

\section{Descriptores}

Procedimientos quirúrgicos; Perfil de Salud; Enfermería pediátrica

\section{Como citar:}

Pan R, Tripode FA, Zullo SA, Silva JL, Szymaniak NP. Perfil de crianças em idade escolar submetidas a cirurgia em um hospital público. Rev Soc Bras Enferm Ped. 2021;21(2):119-24.

\footnotetext{
${ }^{1}$ Universidade Federal do Triângulo Mineiro, Uberaba, MG, Brasil.

Conflitos de interesse: nada a declarar.

Submetido: 20 de Agosto de 2021 | Aceito: 3 de Dezembro de 2021

Autor correspondente: Raquel Pan | E-mail: raquel.pan@uftm.edu.br

DOI: http://dx.doi.org/10.31508/1676-379320210017
} 


\section{Introdução}

Uma intervenção cirúrgica traz situações inusitadas para a criança, como a presença de pessoas estranhas, injeções, ambiente desconhecido e procedimentos dolorosos, o que contribui para reações de insegurança e medo. Por isso, é importante que a criança seja preparada para que os processos psicológicos desencadeados pela situação não comprometam a sua recuperação. ${ }^{(1)}$

O período pré-operatório pode provocar alto grau de ansiedade, em razão do sofrimento do paciente, se esse estiver acometido por doença, ou devido a outros fatores, como o contato direto com outras pessoas que se encontram no mesmo ambiente de espera, a submissão aos exames complementares, a separação de sua família e ambiente, além de outras eventualidades. ${ }^{(1)}$ Sendo assim, é importante reduzir o estresse pré-operatório e da própria hospitalização dessa criança visando a efetiva recuperação pós-operatória. ${ }^{(2)}$

Os profissionais de saúde, principalmente a equipe de enfermagem pediátrica, por ter maior proximidade durante o cuidado à criança, precisam reconhecer e valorizar a relação da criança e sua família. Dessa forma, o papel da equipe não deve se restringir aos procedimentos técnicos, mas atender também a família e suas dúvidas, apoiar iniciativas e oferecer estímulo no desenvolvimento dos cuidados relacionados à criança, mantendo em vista o contexto físico, socioeconômico, cultural e espiritual da família. ${ }^{(3)}$ Tais aspectos mostram a necessidade de conhecer o perfil da criança submetida à intervenção cirúrgica e possíveis riscos. Considerar esses fatores passa a ser essencial para o planejamento dos cuidados de enfermagem no pré, intra e pós-operatório. ${ }^{(4)}$

Informações como idade, nível de desenvolvimento, compreensão da criança e da família, tipo de procedimento a ser realizado e experiência prévia de hospitalização auxiliam neste processo, pois permitem traçar um método mais adequado e individualizado para o preparo perioperatório. A finalidade da obtenção dessas informações é possibilitar um melhor preparo para o momento perioperatório e maior compreensão de seus objetivos, seu significado e seu propósito. ${ }^{(5)}$

Descrever o perfil das crianças em idade escolar submetidas a intervenção cirúrgica torna-se relevante não apenas no sentido de caracterizar a população atendida, mas também de contribuir para a avaliação de possível necessidade de melhoria de espaços de cuidado específicos aos pacientes pediátricos que passam por procedimentos cirúrgicos, de forma que possa amenizar o estresse perioperatório, favorecendo a recuperação dos pacientes, diminuindo tempo de internação e melhorando o fluxo hospitalar. Devido à escassez de estudos nesta temática, a caracterização do perfil das crianças em idade escolar na instituição de estudo, em um determinado período, contribui com a literatura da área e torna possível a comparação com outras realidades hospitalares. Partindo de tal pressuposto, este estudo teve como objetivo caracterizar crianças na idade escolar submetidas a cirurgia em um hospital público do interior de Minas Gerais.

\section{Métodos}

Estudo descritivo e retrospectivo de abordagem quantitativa, no qual foram obtidos dados referentes ao atendimento de crianças em idade escolar internadas na Unidade de Atenção à Saúde da Criança e do Adolescente do Hospital de Clínicas da Universidade Federal do Triângulo Mineiro (HC-UFTM), gerenciado pela Empresa Brasileira de Serviços Hospitalares (Ebserh). Os dados foram disponibilizados pelo Setor de Gestão de Processos e Tecnologia da Informação (SGPTI) do referido hospital. Foram analisados os registros com os seguintes critérios de inclusão: crianças com idade entre 6 e 12 anos de idade e que realizaram procedimento cirúrgico no Centro Cirúrgico do HC-UFTM no período de janeiro de 2017 a dezembro de 2019. Foram excluídos os dados de crianças que realizaram cirurgia em outro serviço ou cidade e ficaram internados no hospital para fazerem o pós-operatório.

A unidade de internação pediátrica conta com 24 leitos, destinados aos egressos da Unidade de Terapia Intensiva (UTI), do Pronto Socorro, da Hematopediatria e das cirurgias eletivas, sendo que alguns desses leitos são ocupados por pacientes de longa permanência. ${ }^{(6)} \mathrm{O}$ hospital onde foi realizado o estudo é o único da região que oferece atendimento de alta complexidade exclusivamente pelo Sistema Único de Saúde (SUS). ${ }^{(6)}$ Este estudo segue a definição de criança em idade escolar dos Descritores em Ciências da Saúde, que é "pessoa de 6 a 12 anos de idade". ${ }^{(7)}$ 
As variáveis estudadas foram: sexo (feminino, masculino, ignorado); idade (6, 7, 8, 9, 10, 11 e 12 anos); número de cirurgias realizadas por paciente; 400 tipos de procedimentos cirúrgicos realizados, assim como natureza (eletiva, urgência/emergência); especialidade (Anestesiologia, Cirurgia buco-maxilo-facial, Cirurgia de cabeça e pescoço, Cirurgia do aparelho digestivo, Cirurgia geral, Cirurgia Pediátrica, Cirurgia Plástica, Cirurgia Torácica, Endoscopia, Ginecologia e Obstetrícia, Hematologia Pediátrica, Neurocirurgia, Odontologia Hospitalar, Oftalmologia, Ortopedia e Traumatologia, Otorrinolaringologia, Urologia); município de residência (munícipio do hospital de estudo, municípios próximos, municípios intermediários, municípios distantes); ano $(2017,2018,2019)$ e mês (janeiro, fevereiro, março, abril, maio, junho, julho, agosto, setembro, outubro, novembro, dezembro) de realização das cirurgias; e destino após cirurgia (domicílio, enfermaria, Sala de Recuperação Anestésica, Unidade de Terapia Intensiva).

A variável número de cirurgias realizadas por paciente foi tratada como quantitativa. As variáveis ano e mês, obtidas a partir da data de realização da cirurgia, assim como a idade do paciente (em anos), foram consideradas como variáveis categóricas, apesar de sua representação numérica. Para as variáveis sexo, tipos de procedimentos cirúrgicos realizados, natureza, especialidade e destino após a cirurgia foram respeitadas as categorias cadastradas no Aplicativo de Gestão para Hospitais Universitários (AGHU). Apenas para a variável destino após a cirurgia foi feito o agrupamento das categorias UTI Geral e UTI Neo-infantil em Unidade de Terapia Intensiva.

Para a descrição da variável residência, os critérios para considerar a distância ao centro de atendimento (Uberaba, MG, Brasil) foram os municípios: próximos, intermediários e distantes. Considerou-se como próximos os municípios com distância igual ou menor a $100 \mathrm{~km}$; intermediários, maior que $100 \mathrm{~km}$ e menor que $500 \mathrm{~km}$; e distantes, maior que $500 \mathrm{~km}$ (Google Maps). ${ }^{(8)}$

O estudo foi aprovado no Comitê de Ética em Pesquisa do HC-UFTM (CAAE 26262519.1.0000.8667) e seguiu os preceitos éticos contidos na Resolução 466/12. ${ }^{(9)}$ Foi solicitada a dispensa do Termo de Consentimento Livre Esclarecido por se tratar de dados secundários.

Os dados fornecidos em formato Microsoft Excel 2016 pela SGPTI foram gerados a partir do AGHU.
A organização dos dados foi realizada por meio do software estatístico Statistica versão 13. Foram geradas tabelas que expressam as distribuições de frequências absolutas e relativas para todas as variáveis estudadas. Também foram construídas tabelas de contingência para verificar a distribuição dos escolares submetidos à cirurgia por sexo e idade; do número de cirurgias realizadas por mês e ano; e da natureza das cirurgias realizadas por ano de ocorrência.

\section{Resultados}

Foram identificados 825 pacientes pediátricos em idade escolar que passaram por procedimentos cirúrgicos entre os anos de 2017 a 2019. Nesse período, ocorreram 1.127 cirurgias ou procedimentos similares, sendo 400 tipos de procedimentos diferentes dentre 17 especialidades. A especialidade com maior número de procedimentos cirúrgicos foi Ortopedia e Traumatologia, com total de 387 (34,2\%) cirurgias (Tabela 1).

Tabela 1. Especialidades cirúrgicas ou procedimentos similares realizados em crianças na idade escolar

\begin{tabular}{lc}
\hline Especialidade & $\mathrm{n}(\%)$ \\
\hline Ortopedia e traumatologia & $387(34,2)$ \\
Cirurgia pediátrica & $204(18,1)$ \\
Cirurgia plástica & $138(12,2)$ \\
Urologia & $118(10,5)$ \\
Oftalmologia & $69(6,1)$ \\
Otorrinolaringologia & $64(5,7)$ \\
Endoscopia & $60(5,3)$ \\
Cirurgia geral & $21(1,9)$ \\
Neurocirurgia & $21(1,9)$ \\
Hematologia pediátrica & $19(1,7)$ \\
Ginecologia e obstetrícia & $9(0,8)$ \\
Cirurgia torácica & $7(0,6)$ \\
Anestesiologia & $3(0,3)$ \\
Cirurgia de cabeça e pescoço & $2(0,2)$ \\
Cirurgia do aparelho digestivo & $2(0,2)$ \\
Odontologia hospitalar & $2(0,2)$ \\
Cirurgia buco-maxilo-facial & $1(0,1)$ \\
Total & $1127(100,0)$ \\
\hline
\end{tabular}

Os cinco tipos de procedimentos cirúrgicos mais realizados foram: endoscopia digestiva alta (59 vezes; $5,2 \%$ ); apendicectomia (40 vezes; $3,5 \%$ ); curativo em grande queimado com ou sem debridamento (39 vezes; $3,5 \%$ ); curativo grau 2 com ou sem desbridamen- 
to (37 vezes; $3,3 \%$ ); e postectomia (29 vezes; $2,6 \%$ ).

Nos três anos, o número de cirurgias variou de 333 a 401. Entre essas, 550 (48,8\%) classificadas como eletivas e 577 (51,2\%) de urgência ou emergência. Foram 825 crianças operadas e 1.127 procedimentos, sendo que 124 pacientes $(15,0 \%)$ foram submetidos a mais de uma cirurgia no período. O número de cirurgias a que esses pacientes foram submetidos variou entre 2 e 19, com exceção de um paciente do sexo masculino, grande queimado, que foi submetido a 50 cirurgias nesse período (Tabela 2).

Tabela 2. Natureza e ano das cirurgias e dos procedimentos similares realizados nos escolares

\begin{tabular}{lccc}
\hline Ano & \multicolumn{2}{c}{ Natureza } & Total \\
& Eletiva & $\begin{array}{c}\text { Urgência/ } \\
\text { Emergência } \\
\mathrm{n}(\%)\end{array}$ & $\mathrm{n}(\%)$ \\
\hline 2017 & $161(48,3)$ & $172(51,7)$ & $333(29,5)$ \\
2018 & $184(45,9)$ & $217(54,1)$ & $401(35,6)$ \\
2019 & $205(52,2)$ & $188(47,8)$ & $393(34,9)$ \\
Total & $550(48,8)$ & $577(51,2)$ & $1127(100,0)$ \\
\hline
\end{tabular}

Em relação ao sexo das crianças, 549 (66,6\%) eram do sexo masculino e 275 (33,3\%) feminino e um paciente não especificado $(0,1 \%)$. A idade mais frequente foi sete anos. Para o sexo feminino, a maior frequência foi verificada nas idades de seis e sete anos (49; $17,8 \%$ cada idade), enquanto para o sexo masculino o maior número de cirurgias ocorreu na idade de oito anos (88; $16,0 \%$ ), conforme a tabela 3 .

Tabela 3. Idade e sexo dos escolares submetidos a cirurgia em um hospital público no interior de Minas Gerais

\begin{tabular}{lcccc}
\hline $\begin{array}{l}\text { Idade } \\
\text { (anos) }\end{array}$ & $\mathrm{n}(\%)$ & $\begin{array}{c}\text { Feminino } \\
\mathrm{n}(\%)\end{array}$ & $\begin{array}{c}\text { Sexo } \\
\text { Masculino } \\
\mathrm{n}(\%)\end{array}$ & $\begin{array}{c}\text { Ignorado } \\
\mathrm{n}(\%)\end{array}$ \\
\hline 6 & $124(15,0)$ & $49(17,8)$ & $75(13,7)$ & - \\
7 & $131(15,9)$ & $49(17,8)$ & $82(14,9)$ & - \\
8 & $120(14,5)$ & $32(11,6)$ & $88(16,0)$ & - \\
9 & $119(14,4)$ & $40(14,5)$ & $79(14,4)$ & - \\
10 & $103(12,6)$ & $33(12,0)$ & $69(12,6)$ & $1(100,0)$ \\
11 & $120(14,5)$ & $42(15,3)$ & $78(14,2)$ & - \\
12 & $108(13,1)$ & $30(11,0)$ & $78(14,2)$ & - \\
Total & $825(100,0)$ & $275(33,3)$ & $549(66,6)$ & $1(0,1)$ \\
\hline
\end{tabular}

A maioria das crianças era residente do próprio município de Uberaba-MG (475; 57,6\%), como demonstrado na tabela 4.

No pós-operatório imediato, na maioria das cirurgias $(1.115 ; 98,9 \%)$ as crianças foram encaminhadas di-
Tabela 4. Residência dos escolares submetidos à cirurgia, em um hospital público do interior de Minas Gerais

\begin{tabular}{|c|c|c|}
\hline & & $n(\%)$ \\
\hline $\begin{array}{l}\text { Município do } \\
\text { hospital de } \\
\text { estudo }\end{array}$ & Uberaba-MG & $475(57,6)$ \\
\hline $\begin{array}{l}\text { Municípios } \\
\text { próximos }\end{array}$ & $\begin{array}{l}\text { Água Comprida, Campo Florido, Conceição } \\
\text { das Alagoas, Conquista, Delta, Jubaí, } \\
\text { Pirajuba, Sacramento, Santa Juliana e } \\
\text { Veríssimo-MG }\end{array}$ & $119(14,4)$ \\
\hline $\begin{array}{l}\text { Municípios } \\
\text { intermediários }\end{array}$ & $\begin{array}{l}\text { Aparecida de Minas, Araguari, Araxá, } \\
\text { Campos Altos, Carmo de Paranaíba, } \\
\text { Carneirinho, Comendador Gomes, Fronteira, } \\
\text { Frutal, Ibiá, Itapagipe, Ituiutaba, Iturama, } \\
\text { Lagoa Formosa, Lagoa Grande, Patos de } \\
\text { Minas, Pedrinópolis, Perdizes, Planura, } \\
\text { Prata, Pratinha, São Francisco, São } \\
\text { Francisco de Sales, São Gotardo, Tapira, } \\
\text { Unaí, União de Minas-MG e Itumbiara - Go }\end{array}$ & $221(26,8)$ \\
\hline $\begin{array}{l}\text { Municípios } \\
\text { distantes }\end{array}$ & $\begin{array}{l}\text { Arinos, Cabeceira Grande, Capelinha, Ibiaí, } \\
\text { Brasília-DF } \\
\text { Grajaú-MA } \\
\text { São Francisco de Sales-PR }\end{array}$ & $10(1,2)$ \\
\hline Total & & $825(100,0)$ \\
\hline
\end{tabular}

retamente à Sala de Recuperação Anestésica da instituição. Em outros casos, as crianças foram diretamente para o domicílio $(5 ; 0,4 \%)$ ou enfermaria $(3 ; 0,3 \%)$. Alguns considerados graves, tiveram encaminhamento à Unidade de Terapia Intensiva (4; $0,4 \%)$. Os meses com maior número de cirurgias foram maio $(124 ; 11,0 \%)$, setembro $(117 ; 10,4 \%)$ e outubro $(119 ; 10,6 \%)$, de acordo com a tabela 5 .

Tabela 5. Número por mês e dia de cirurgias realizadas em escolares

\begin{tabular}{|c|c|c|c|c|c|}
\hline Meses & $\begin{array}{l}2017 \\
n(\%)\end{array}$ & $\begin{array}{l}2018 \\
\mathrm{n}(\%)\end{array}$ & $\begin{array}{l}2019 \\
\mathrm{n}(\%)\end{array}$ & $\begin{array}{l}\text { Total } \\
\mathrm{n}(\%)\end{array}$ & $\begin{array}{c}\text { Cirurgia/ } \\
\text { dia }\end{array}$ \\
\hline Janeiro & $21(6,3)$ & $32(8,0)$ & $18(4,6)$ & $71(6,3)$ & 0,76 \\
\hline Fevereiro & $22(6,6)$ & $23(5,7)$ & $19(4,8)$ & $64(5,8)$ & 0,76 \\
\hline Março & $35(10,5)$ & $35(8,7)$ & $24(6,1)$ & $94(8,3)$ & 1,01 \\
\hline Abril & $22(6,6)$ & $41(10,2)$ & $32(8,1)$ & $95(8,4)$ & 1,06 \\
\hline Maio & $21(6,3)$ & $45(11,3)$ & $58(14,8)$ & $124(11,0)$ & 1,33 \\
\hline Junho & $26(7,8)$ & $37(9,2)$ & $41(10,4)$ & $104(9,2)$ & 1,16 \\
\hline Julho & $23(6,9)$ & $23(5,7)$ & $32(8,1)$ & $78(6,9)$ & 0,84 \\
\hline Agosto & $48(14,4)$ & $22(5,5)$ & $34(8,7)$ & $104(9,2)$ & 1,12 \\
\hline Setembro & $34(10,2)$ & $34(8,5)$ & $49(12,5)$ & $117(10,4)$ & 1,30 \\
\hline Outubro & $31(9,3)$ & $45(11,3)$ & $43(10,9)$ & $119(10,6)$ & 1,28 \\
\hline Novembro & $17(5,2)$ & $35(8,7)$ & $27(6,9)$ & $79(7,0)$ & 0,88 \\
\hline Dezembro & $33(9,9)$ & $29(7,2)$ & $16(4,1)$ & $78(6,9)$ & 0,84 \\
\hline Total & $333(29,5)$ & $401(35,6)$ & $393(34,9)$ & $1.127(100,0)$ & 1,03 \\
\hline
\end{tabular}

\section{Discussão}

Os achados desde estudo corroboram com a pesquisa desenvolvida na região Sul do Brasil que constatou 
que a maioria das crianças internadas para tratamento cirúrgico eram do sexo masculino e estavam em idade escolar. ${ }^{(10)}$ A maioria dos pacientes serem do sexo masculino também está em conformidade com outro estudo. ${ }^{(11)}$ Esse achado pode estar relacionado devido ao maior número de procedimentos ser da especialidade de Ortopedia e Traumatologia. As brincadeiras infantis costumam ter relação com gênero, visto que crianças do sexo masculino têm maior possibilidade de estarem envolvidas em brincadeiras com maior risco de acidentes e fraturas. Além disso, envolvem fatores socioculturais, normalmente meninos praticam atividades mais dinâmicas, apresentando maior risco de lesões, enquanto as meninas costumam ser mais vigiadas e com menos liberdade. ${ }^{(12)}$

O número significativo de cirurgias de urgência e o fato da especialidade de Ortopedia e Traumatologia ser a com maior número de procedimentos também ocorrem devido ao hospital em questão ser referência de urgência e emergência e o único que oferece atendimento de alta complexidade na região, atendendo 27 municípios. ${ }^{(6)} \mathrm{Na}$ literatura, outros estudos realizados em Hospitais de referência identificaram com mais frequência demandas cirúrgicas. ${ }^{(11)}$

Quanto ao número de crianças que foram submetidas a mais de uma cirurgia $(124 ; 15 \%)$, situação semelhante foi encontrada em outro estudo realizado em um hospital pediátrico no município do Rio de Janeiro, em que do total de crianças submetidas a intervenções cirúrgicas no período estudado 10 (7\%) passaram por mais de uma cirurgia. ${ }^{(4)}$ Ser submetida a uma intervenção cirúrgica pode ser considerada uma experiência traumática para criança. Portanto, passar por mais de um procedimento cirúrgico requer ainda mais cuidado e atenção por parte da equipe, bem como apoio familiar. As orientações à criança e família devem fazer parte do plano de cuidados, além da realização de técnicas e procedimentos. ${ }^{(10)}$

Com relação à residência, a maioria dos pacientes era de Uberaba ou de cidades próximas. Porém, se tem um número significativo de cidades de distância intermediária ou distante. Isso representa um número alto de pacientes e famílias que têm que se deslocar a outra cidade durante o período de internação. A distância pode apresentar uma dificuldade, pois, durante esse período, a família tem seus gastos aumentados, devido a despesas com locomoção, alimentação, en- tre outros. ${ }^{(13)}$ Ademais, se houver necessidade de um maior tempo de internação, isso pode gerar mudanças na esfera familiar resultantes da necessidade de acompanhamento a um filho no hospital e da participação nos seus cuidados em tempo integral. ${ }^{(14)}$

Em cirurgias de emergência também deve-se levar em consideração que devido a sua natureza imediata, a criança e a família estão em uma situação inesperada que pode acarretar estresse e ansiedade, característica não tão presente em cirurgias eletivas. Destaca-se ainda a relevância do cuidado de enfermagem voltado às necessidades da criança no perioperatório que pode envolver internação prolongada, além do estresse e da ansiedade já mencionados. Nesse sentido, vale a pena ressaltar que hospitalizações podem ser evitáveis ${ }^{(15,16)}$ quando se lança mão de orientações e campanhas de prevenção de acidentes na infância em escolas, bem como ações educativas em sala de espera.

A presente pesquisa apresenta como limitação a não obtenção de dados que seriam de relevância para discussão do objeto de estudo, como tipo de anestesia, desfecho (alta, óbito ou transferência) e dia da semana que a cirurgia foi realizada, por ausência nos registros hospitalares. Por se tratar de um estudo que busca caracterizar crianças em idade escolar submetidas à cirurgia, $\mathrm{o}$ conhecimento desse último dado se faz importante para verificar se a instituição visa agendar as cirurgias eletivas em período de férias escolares ou finais de semana e feriado; bem como se existe preocupação com a continuidade das atividades escolares em caso de internação prolongada. Outra limitação é o fato de que o hospital não possuía um sistema que reunisse todas as informações de prontuários até meados de 2014, quando teve início o sistema AGHU. Os dados disponibilizados referentes aos anos de 2015 e 2016 estavam incompletos, limitando os achados deste estudo a partir do ano de 2017.

\section{Conclusão}

A caracterização das crianças em idade escolar submetidas à intervenção cirúrgica em um hospital público do interior de Minas Gerais demonstra um perfil sociodemográfico mais frequente em crianças com menor idade e do sexo masculino, submetidas a procedimento cirúrgico da especialidade de Ortopedia e Traumatologia, especialmente em urgência e emergência. 
Este estudo possibilitou conhecer a população infantil atendida, a fim de contribuir para a organização e o planejamento da assistência prestada às crianças em tratamento cirúrgico, além de avaliar a necessidade de melhoria de espaços de cuidado específicos aos pacientes pediátricos que passam por procedimentos cirúrgicos, para que dessa forma amenize o estresse e a ansiedade perioperatórios, podendo assim colaborar na recuperação e diminuindo tempo de internação.

\section{Contribuições}

Pan R, Tripode FA, Zullo AS, Silva JLS e Szymaniak NP contribuíram com a concepção do projeto, análise e interpretação dos dados, redação do artigo, revisão crítica relevante do conteúdo intelectual e aprovação da versão final a ser publicada.

\section{Referências}

1. Broering $C$, Crepaldi M. Percepções e informações das mães sobre a cirurgia de seus filhos. Fractal. 2018; 30(1):3-11.

2. Broering CV, Souza CD, Kaszubowski E, Crepaldi MA. Efectos de preparaciones psicológicas prequirúrgicas sobre el estrés y la ansiedad de niños y niñas. Acta Colomb Psicol. 2017; 21(1):214-248.

3. Prado $\mathrm{P}$, Cardoso N, Souza A, Figueiredo M. Vivenciando o processo cirúrgico: percepção e sentimentos da criança. Rev Baiana Enferm. 2017; 31(3):e17648.
4. Santos QF, Góes FG, Silva AC, Pereira FM, Ferraz AN, Vollmer RB. Perfil de pacientes submetidos à cirurgia geral em um hospital pediátrico: implicações para a enfermagem. Rev Enferm Atual In Derme. 2019;87(25):1-9.

5. Broering C, Crepaldi M. Preparação psicológica e 0 estresse de crianças submetidas a cirurgias. Psicol Estud. 2011; 16(1):15-23.

6. Empresa Brasileira de Serviços Hospitalares (Ebserh). Hospital de Clínicas da UFTM. Carta de Serviços ao Cidadão HC - UFTM. Uberaba: Hospital de Clínicas da Universidade Federal do Triângulo Mineiro; 2017 [acesso em 25 set 2019]. Disponível em: http://www2.ebserh. gov.br/web/hc-uftm/historia

7. Biblioteca Virtual em Saúde (BVS). Descritores em Ciências da Saúde. São Paulo: BVS; 2017 [citado 2020 Out 10]. Disponível em: https://decs.bvsalud.org/ths/ resource/?id=2694\&filter=ths_exact_term\&q=crian\%C3\%A7a

8. Google Maps. 2020 [acesso em 14 out 2020]. Disponível em: https://www.google.com.br/ maps/

9. Brasil. Ministério da Saúde. Conselho Nacional de Saúde. Resolução No. 466 de 12 de dezembro de 2010. Brasília (DF): Ministério da Saúde; 2010 [citado 2021 Ago 20]. Disponível em: http://bvsms.saude.gov.br/bvs/saudelegis/cns/2013/ res0466_12_12_2012.html

10. Silveira A, Neves E, Famoso AF, Donaduzzi JC, Junges CF, Zamberlan KC. Caracterização de crianças em tratamento cirúrgico em um hospital escola no Sul do Brasil. Rev Enferm UFSM. 2011;1(2):174-82.

11. Alves B, Santos T, Ferrari R, Tacla M, Sant'Anna F, Lopes E. Criança hospitalizada: caracterização dos procedimentos cirúrgicos em um hospital escola público. Semina Cienc Biol Saúde. 2015;36(Supl. 1):317-24.

12. Hernández CM, NúñezVP, Doural KG, Machado AA. Características de crianças hospitalizadas por queimaduras em um hospital em Manzanillo, Cuba. Rev Bras Queimaduras. 2017; 16(3):169-73.

13. Gomes G, Erdmann A, Oliveira P, Xavier D, Santos S, Farias D. The family living the time during the hospitalization of the child: contributions for nursing. Esc Anna Nery. 2014; 18(2):23440.

14. Rodrigues JI, Fernandes SM, Marques GF. Preocupações e necessidades dos pais de crianças hospitalizadas. Saude Soc. 29(2):e190395.

15. Godinho IA. Saúde do trabalhador: atenção primária para hospitalizações evitáveis. LIPH Sci J. 2015;2(2):41-61.

16. Ferreira CM. Infecções parasitárias em crianças: projeto de intervenção para hospitalizações evitáveis. LIPH Sci J. 2015;2(1):13-28. 